\title{
Two-Fluid Nonlinear Theory of Response of Tokamak Plasma to Resonant Magnetic Perturbation
}

\author{
Richard Fitzpatrick \\ Department of Physics \\ Institute for Fusion Studies \\ University of Texas at Austin \\ Austin TX, 78712, USA
}

\begin{abstract}
A comprehensive two-fluid nonlinear theory of magnetic reconnection driven at a single, tearing-stable, rational surface embedded in an H-mode tokamak plasma is presented. The surface is assumed to be resonant with one of the dominant helical harmonics of an applied resonant magnetic perturbation (RMP). The theory described in this paper is highly relevant to the problem of understanding the physics of RMP-induced edge localized mode (ELM) suppression in tokamak plasmas.
\end{abstract}




\section{INTRODUCTION}

Tokamak discharges operating in high-confinement mode (H-mode) $)^{1}$ exhibit intermittent bursts of heat and particle transport, emanating from the outer regions of the plasma, that are known as edge localized modes (ELMs). $\stackrel{2}{2}$ ELMs are fairly benign in present-day tokamaks. However, it is estimated that the heat load that ELMs will deliver to the plasma-facing components in the divertor of a reactor-scale tokamak will be large enough to unacceptably limit the lifetimes of these components via erosion $\underline{3}^{\underline{3}}$

The most promising method for the control of ELMs is via the application of static resonant magnetic perturbations (RMPs). Complete RMP-induced ELM suppression was first demonstrated in the DIII-D tokamak via the application of static, non-axisymmetric, magnetic fields with toroidal mode number $n_{\varphi}=3 \stackrel{4}{ }$ Subsequently, either mitigation or compete suppression of ELMs has been demonstrated on the JET, $\underline{5}$ ASDEX-U,,$\underline{6}$ KSTAR,,$\underline{7}$ and EAST $\stackrel{8}{*}$ tokamaks. Furthermore, the parameter range over which ELM suppression has been observed in DIII-D has been extended to include low-collisionality ITER-shaped

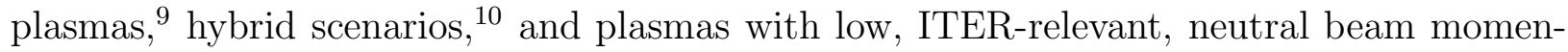
tum injection. 11 These achievements have led to increased confidence that the RMP ELM control technique can operate effectively in ITER.$^{12}$

At present, the physical mechanism of RMP-induced ELM suppression is not fully understood. ELMs are generally thought to be caused by peeling-ballooning instabilities with intermediate toroidal mode numbers $\left(3<n_{\varphi}<20\right)$ that are driven by the strong pressure gradients and current densities characteristic of the edge region of an H-mode discharge, which is known as the pedestal. 13 Consequently, early attempts to understand RMP-induced ELM suppression focused on the role of RMPs in reducing the pressure gradient, and, thereby, reducing the bootstrap current density, in the pedestal. In particular, the initial observations of ELM suppression were interpreted as an indication that the magnetic field in the pedestal had been rendered stochastic by the applied RMP, leading to greatly enhanced transport via thermal diffusion along magnetic field-lines. $\stackrel{4}{\underline{4}} \underline{4}$ This simplistic explanation was quickly abandoned because no reduction in the electron temperature gradient in the pedestal is observed during RMP ELM suppression experiments, whereas a very significant reduction would be expected in the presence of stochastic fields. It is now generally accepted that response currents generated within the plasma play a crucial role in the perturbed equilibrium 
in the presence of RMPs, and that these currents act to prevent the formation of magnetic islands - a process known as shielding - and, thereby, significantly reduce the stochasticity of the magnetic field. $\frac{15}{\underline{5}}$

Current thinking suggests that density and temperature gradients in the pedestal are fixed by stiff transport due to high- $n_{\varphi}$ instabilities, and that the pedestal grows in radial extent, characterized by these fixed gradients, until the peeling/ballooning stability threshold is reached, and an ELM is triggered. $\stackrel{16}{*}$ According to this scenario, ELM suppression is achieved by limiting the expansion of the pedestal before the peeling/ballooning stability threshold is crossed. $\underline{\underline{17}}$ It is hypothesized that pedestal expansion is halted by a region of enhanced transport at the top of the pedestal that is not suppressed by $\mathbf{E} \times \mathbf{B}$ velocity shear, and that this region is associated with the presence of an RMP-driven magnetic island chain located just outside, but close to the top of, the pedestal. $\underline{18}^{18}$

The aim of this paper is to develop a physical understanding of the dynamics of magnetic reconnection driven at a single rational surface that is resonant with one of the dominant helical harmonics of an applied RMP. The rational surface is assumed to be located just outside, but close to the top of, the pedestal of an H-mode tokamak plasma. The rational surface is also assumed to be intrinsically tearing-stable, so that any magnetic reconnection that occurs at the surface is due to the action of the applied RMP. Dealing with a single rational surface is a reasonable approach because the aforementioned lack of magnetic stochasticity in the pedestal indicates that any magnetic island chains driven in this region are sufficiently narrow that they do not overlap with one another, which suggests that they evolve independently, at least to a first approximation. We are also concentrating on a particular rational surface (i.e., one located close to the top of the pedestal, and resonant with a dominant RMP harmonic) because there is ample evidence from experimental $q_{95}$ scans that RMP-induced ELM suppression depends crucially on the existence of this surface. $\underline{6} \underline{\underline{9}} \cdot \underline{18}$

This paper does not explicitly address the mechanism by which driven reconnection at the aforementioned rational surface leads to ELM suppression. However, there are two obvious candidate mechanisms. The first is the degradation of radial confinement due to the fact that heat and particles can flow around the magnetic separatrix from one side of the island chain to the other $\underline{\underline{19}}$ The second is the modification of the local plasma flow induced by the electromagnetic locking torque exerted on the island chain by the RMP. (A change in the flow affects the local $\mathbf{E} \times \mathbf{B}$ velocity shear which, in turn, can modify the local transport.) 
However, it is clear that both of these mechanisms are only operative if a relatively wide island chain is driven at the rational surface. In other words, they only work effectively if strong shielding breaks down at the rational surface. Hence, this paper will investigate the circumstances in which the breakdown of shielding occurs.

Over the years, many different theoretical approaches have been taken to investigate driven reconnection at an intrinsically stable rational surface embedded in a tokamak plasma. These include single-fluid linear response models,,$\underline{20-22}$ two-fluid linear response models, $\underline{23-25}$ kinetic linear response models, $\stackrel{26}{\underline{a}}$ single-fluid nonlinear response models, $\stackrel{21}{\underline{a}}$ and two-fluid nonlinear response models. $\frac{27}{\underline{*}}$

Generally speaking, the aforementioned response models predict the same phenomenology. (Here it is assumed that the linear models are used to calculate a quasi-linear electromagnetic locking torque that is balanced against a viscous restoring torque.) There exists a shielded state in which driven magnetic reconnection at the rational surface is strongly suppressed by plasma flow, and a penetrated state in which the plasma flow is modified in such a manner as to allow significant magnetic reconnection. As the resonant component of the RMP is gradually ramped up, a bifurcation from the shielded to the penetrated state is triggered once the resonant component exceeds a critical penetration threshold. If the resonant component is then gradually ramped down then a bifurcation from the penetrated to the shielded state is triggered once the resonant component falls below a critical de-penetration threshold. However, the de-penetration threshold is usually significantly smaller than the penetration threshold. Both bifurcations are accompanied by sudden changes in the plasma flow at the rational surface, which are, in turn, induced by changes in the electromagnetic locking torque exerted in the vicinity of the surface by the resonant component of the RMP.

Despite the discussion in the previous paragraph, it is important to appreciate that there exist significant differences between the predictions of the various response models. Singlefluid linear and single-fluid nonlinear models predict that the penetrated state is achieved when the $\mathbf{E} \times \mathbf{B}$ velocity is reduced to zero at the rational surface. ${ }^{20,21}$ On the other hand, two-fluid linear theories predict that the penetrated state is achieved when the perpendicular electron fluid velocity is reduced to zero at the rational surface. ${ }^{24}$ Finally, two-fluid nonlinear theories predict that the penetrated state is achieved when a velocity that is offset from the $\mathbf{E} \times \mathbf{B}$ velocity in the ion or electron diamagnetic direction, depending on the local values of $\eta_{i}$ and $Z_{\text {eff }}$, is reduced to zero at the rational surface. 27 (See Sect. IID.) There are also marked 
differences in the predicted dependences of the shielding factors, penetration thresholds, and de-penetration thresholds, on plasma parameters between the various models.

It is clearly important to choose the correct response model when investigating driven magnetic reconnection in a tokamak plasma. But, what is the appropriate model for the problem under investigation? It is the thesis of this paper that the correct model is a twofluid nonlinear model, and that all of the other models mentioned previously are either inadequate or invalid. It is obvious that a single-fluid model is inadequate, given the significant differences that exist between, for example, the $\mathbf{E} \times \mathbf{B}$ velocity and the perpendicular electron fluid velocity in a typical H-mode pedestal. By definition, a linear model becomes invalid as soon as the width of the magnetic separatrix exceeds the layer width. Careful comparisons performed in Ref. 28 reveal that in typical $n=2$ DIII-D ELM suppression experiments the driven island width at the $8 / 2$ rational surface greatly exceeds the linear layer width in the penetrated state (which is hardly surprising). However, the driven island width also exceeds the linear layer width in the shielded state. This suggests that both the penetrated and shielded states are governed by nonlinear physics. There are other strong indications of nonlinear behavior in RMP ELM suppression experiments. For instance, Fig. 5 in Ref. 29 shows data from an $n=2$ ELM suppression experiment performed on DIII-D in which a bifurcation from a penetrated to a shielded state takes place. This transition is accompanied by the "spin-up" of the locked magnetic island chain associated with the penetrated state. Such behavior is impossible within the context of linear response theory, but is easily explained within the context of nonlinear theory. (See Sect. IVD, Furthermore, Fig. 29 in Ref. 30 shows a shielded state in an $n=3$ RMP suppression experiment performed on DIII-D with a fixed RMP amplitude in an otherwise steady-state plasma. It can be seen that the state seems to consist of island chains of pulsating width, driven at different rational surfaces in the pedestal, and rotating in highly uneven manners. Moreover, the electromagnetic torque exerted on the plasma is time-varying, as evidenced by the time-varying ion toroidal velocity. As before, all of these behaviors are impossible within the context of linear response theory, but are easily accounted for within the context of nonlinear theory. $\underline{\underline{27}}$ (See Sect. IVC)

The remainder of this paper is devoted to the exposition of a two-fluid nonlinear theory of driven magnetic reconnection at a single, tearing-stable, rational surface embedded in a tokamak plasma. As explained in the previous discussion, such a theory is relevant to the 
problem of understanding RMP-induced ELM suppression.

\section{TWO-FLUID NONLINEAR RESPONSE MODEL}

\section{A. Introduction}

This section describes the particular two-fluid nonlinear response model of driven magnetic reconnection at a single, tearing-stable, rational surface, embedded in a tokamak plasma, that is adopted in this paper.

The model in question was derived in Ref. 27. The core of the model is a single-helicity version of the well-known four-field model of Hazeltine, Kotschenreuther, and Morrison. ${ }^{31}$ The core model is augmented by phenomenological terms representing anomalous cross-field particle and momentum transport due to small-scale plasma turbulence. Finally, the model includes approximate (i.e., flux-surface averaged) expressions for the divergences of the neoclassical stress tensors. These expressions allow neoclassical ion poloidal and perpendicular flow damping to be incorporated into the analysis.

In Ref. 27, an ordering scheme is adopted that is suitable for a constant- $\psi \stackrel{32}{ }$, sonic, $\stackrel{33}{\text { mag- }}$ netic island chain whose radial width is similar to the ion poloidal gyroradius. Momentum transport in the island region is assumed to be dominated by poloidal and perpendicular ion neoclassical flow damping (rather than perpendicular ion viscosity). After a great deal of analysis, the formalism reduces to a nonlinear island width evolution equation and a nonlinear island phase evolution equation. (See Sect. IIF.) These two equations are coupled together. The formalism also determines the density, temperature, and flow profiles in the island region. (See Sects. IG and IH.)

\section{B. Magnetic Island Chain}

Consider a large aspect-ratio, low- $\beta$, circular cross-section, tokamak plasma equilibrium of major radius $R_{0}$, and toroidal magnetic field-strength $B_{0}$. Let us adopt a right-handed, quasi-cylindrical, toroidal coordinate system $(r, \theta, \varphi)$ whose symmetry axis $(r=0)$ coincides with the magnetic axis. The coordinate $r$ also serves as a label for the unperturbed (by the island chain) magnetic flux-surfaces. Let the equilibrium toroidal magnetic field and toroidal plasma current both run in the $+\varphi$ direction. 
Suppose that a helical magnetic island chain (driven by an RMP), with $m_{\theta}$ poloidal periods, and $n_{\varphi}$ toroidal periods, is embedded in the aforementioned plasma. The island chain is assumed to be radially localized in the vicinity of its associated rational surface, minor radius $r_{s}$, which is defined as the unperturbed magnetic flux-surface at which $q\left(r_{s}\right)=$ $m_{\theta} / n_{\varphi}$. Here, $q(r)$ is the equilibrium safety-factor profile. Let the full radial width of the island chain's magnetic separatrix be $4 w$. In the following, it is assumed that $r_{s} / R_{0} \ll 1$ and $w / r_{s} \ll 1$.

The magnetic flux surfaces in the island region correspond to the contours of $\underline{27}$

$$
\Omega(X, \zeta)=\frac{1}{2} X^{2}+\cos \zeta,
$$

where $X=\left(r-r_{s}\right) / w, \zeta=\chi-\phi_{p}(t)$, and $\chi=m_{\theta} \theta-n_{\varphi} \varphi$. The O-points of the island chain are located at $X=0$ and $\zeta=\pi$, whereas the X-points are located at $X=0$ and $\zeta=0$. The magnetic separatrix corresponds to $\Omega=1$, the region enclosed by the separatrix to $-1 \leq \Omega<1$, and the region outside the separatrix to $\Omega>1$.

It is helpful to define the concept of a vacuum island chain, which is is defined as the static island chain obtained by naively superimposing the vacuum resonant component of the RMP onto the unperturbed plasma equilibrium. Let $4 w_{v}$ be the full radial width of the vacuum island chain. Moreover, $\phi_{p}$ is conveniently defined as the helical phase shift between the true and the vacuum island chains.

\section{Neoclassical Flow Damping}

Let $\nu_{\theta i}$ and $\nu_{\perp i}$ be the neoclassical ion poloidal and perpendicular flow damping rates, respectively, in the vicinity of the island chain. The relationships between these damping rates and the assumed forms of the neoclassical stress tensors are specified in Ref. 34 .

Neoclassical ion poloidal flow damping acts to relax the ion poloidal flow velocity in the vicinity of the rational surface to $-\lambda_{\theta i} \eta_{i} V_{*}$, whereas neoclassical ion perpendicular flow damping acts to relax the ion perpendicular flow velocity in the vicinity of the rational surface to $-\lambda_{\perp i} \eta_{i} V_{*}$. Here, $V_{*}=T_{i 0} /\left(e B_{0} L_{n}\right)$ is the equilibrium ion diamagnetic velocity at the rational surface (due to density gradients only), $T_{i 0}=T_{i}\left(r_{s}\right), e$ is the magnitude of the electron charge, $L_{n}=-1 /\left(d \ln n_{e} / d r\right)_{r=r_{s}}$, and $\eta_{i}=\left(\ln T_{i} / d \ln n_{e}\right)_{r=r_{s}}$. Furthermore, $T_{i}(r)$ is the equilibrium ion temperature profile, and $n_{e}(r)$ the equilibrium electron number 
density profile. The values of the dimensionless neoclassical velocity parameters, $\lambda_{\theta i}$ and $\lambda_{\perp i}$, are specified in Appendix $\mathrm{A}$.

\section{Natural Frequency}

The natural frequency, $\omega_{0}$, is defined as the propagation frequency (i.e., $d \phi_{p} / d t$ ) that a naturally unstable magnetic island chain, resonant at the rational surface in question, would have in the absence of an RMP. ${ }^{20}$ According to the analysis of Ref. 27,

$$
\omega_{0}=\omega_{E}-\left[1+\left(1-\lambda_{\theta i}\right) \eta_{i}\right] k_{\theta} V_{*}
$$

where $\omega_{E}$ is the equilibrium value of the $\mathbf{E} \times \mathbf{B}$ frequency at the rational surface, and $k_{\theta}=m_{\theta} / r_{s}$. Given that $1-\lambda_{\theta i}$ varies between -0.173 , for a pure electron-hydrogen plasma, and -0.5 , for a very impure electron-hydrogen plasma (see Table I), the previous formula implies that $\omega_{0}-\omega_{E}<0$ unless $\eta_{i}$ exceeds a critical value. This critical value is 5.78 for a pure plasma, and 2 for a very impure plasma. (See Table If) If $\eta_{i}$ does not exceed the critical value then $\omega_{0}-\omega_{E}<0$ : i.e., the natural frequency is offset from the local $\mathbf{E} \times \mathbf{B}$ frequency in the ion diamagnetic direction. On the other hand, if $\eta_{i}$ exceeds the critical value then $\omega_{0}-\omega_{E}>0$ : i.e., the natural frequency is offset from the local equilibrium $\mathbf{E} \times \mathbf{B}$ frequency in the electron diamagnetic direction. Incidentally, the fact that magnetic island chains can propagate in the ion diamagnetic direction relative to the local equilibrium $\mathbf{E} \times \mathbf{B}$ frame has been verified experimentally $\underline{35}, \underline{36}$ Note, finally, that the physics that determines the natural frequency of a nonlinear magnetic island chain is completely different to that which determines the rotation frequency of a linear drift-tearing mode. ${ }^{37}$

The natural frequency plays a crucial role in the theory of driven magnetic reconnection at a tearing-stable rational surface because, in order for strong shielding at the surface to break down, the local plasma flow must be modified in such a manner that the natural frequency becomes zero. (See Sect. IVE.)

\section{E. Fundamental Timescales}

Let

$$
\tau_{H}=\frac{2 \sqrt{I_{1} I_{v}}}{\left(2 m_{\theta}\right)^{3 / 2}}\left(\frac{q_{s}}{\epsilon_{s}}\right)^{2} L_{q}\left(\frac{\mu_{0} n_{0} m_{i}}{B_{0}^{2}}\right)^{1 / 2}
$$


be the effective hydromagnetic timescale at the rational surface. Here, $q_{s}=m_{\theta} / n_{\varphi}, \epsilon_{s}=$ $r_{s} / R_{0}, L_{q}=1 /(d \ln q / d r)_{r=r_{s}}, n_{0}=n_{e}\left(r_{s}\right)$, and $m_{i}$ is the ion mass. The dimensionless quantities $I_{1}$ and $I_{v}$ are defined in Appendix B.

Let

$$
\tau_{R}=\frac{I_{i}}{6 m_{\theta}} \frac{\mu_{0} r_{s}^{2}}{\eta_{\|}}
$$

be the effective resistive diffusion timescale at the rational surface. Here, $\eta_{\|}$is the equilibrium parallel electrical resistivity at the rational surface. The dimensionless quantities $I_{i}$ is defined in Appendix B,

Finally, let

$$
\omega_{D}=\left(\frac{\epsilon_{s}}{q_{s}}\right)^{2} \frac{\nu_{\theta i}}{I_{1}}
$$

be the effective poloidal flow damping rate at the rational surface. Incidentally, according to Ref. 38,

$$
\omega_{D}=\frac{f_{t}}{I_{1}} \nu_{i},
$$

where $f_{t}$ is the fraction of trapped particles, and $\nu_{i}$ is the ion collision frequency. (Both quantities are evaluated at the rational surface.) Here, it is assumed that the ions are in the banana collisionality regime.

\section{F. Island Evolution Equations}

The two-fluid nonlinear response model derived in Ref. 27 reduces to an island width evolution equation,

$$
\lambda_{R} \frac{d \xi}{d T}=-\xi^{2 / 3}+b_{v} \cos \phi_{p},
$$

and an island phase evolution equation,

$$
\frac{d^{2} \phi_{p}}{d T^{2}}+\frac{d \phi_{p}}{d T}+b_{v} \xi^{1 / 3} \sin \phi_{p}=\gamma .
$$


Here,

$$
\begin{aligned}
T & =\omega_{D} t, \\
\lambda_{R} & =S\left(\omega_{D} \tau_{H}\right)^{5 / 3}, \\
S & =\frac{\tau_{R}}{\tau_{H}}, \\
\xi & =\hat{w}^{3}, \\
\hat{w} & =\frac{w}{w_{0}}, \\
\frac{w_{0}}{r_{s}} & =\left(\omega_{D} \tau_{H}\right)^{2 / 3}, \\
b_{v} & =\left(\frac{w_{v}}{w_{0}}\right)^{2}, \\
\gamma & =\frac{\omega_{0}}{\omega_{D}} .
\end{aligned}
$$

In Eqs. (77) and (8), $\xi$ characterizes the island width (actually, it is proportional to the cube of the island width), $\phi_{p}$ is the helical phase of the island chain relative to the vacuum island chain, $b_{v}$ is the normalized amplitude of the resonant component of the RMP, $\lambda_{R}$ is the ratio of the typical island width growth timescale to the typical island rotation timescale, and $\gamma$ is the normalized island natural frequency.

The first term on the right-hand side of Eq. (7) represents the intrinsic stability of the island chain. (Here, it is assumed that $\Delta^{\prime} r_{s}=-2 m_{\theta}$, where $\Delta^{\prime}$ is the conventional tearing stability index. $\stackrel{32}{)}$ The second term represents the effect of the resonant component of the RMP on island width evolution.

The first term on the left-hand side of Eq. (8) represents ion inertia. The second term represents ion neoclassical flow damping. The third term represents the electromagnetic locking torque due to the resonant component of the RMP. Finally, the term on the righthand side represents intrinsic plasma flow at the rational surface.

Equations (77) and (8) are highly nonlinear, and, in general, can only be solved numerically. Numerical integration of these equations is a relatively straightforward task, apart from one caveat. The quantity $\xi$ cannot be negative (because the island width cannot be negative). Hence, when integrating Eqs. (7) and (8), if $\xi$ passes through zero then the 
following transformation is applied: $\underline{\underline{39}}$

$$
\begin{gathered}
\xi \rightarrow-\xi \\
\phi_{p} \rightarrow \phi_{p}+\operatorname{sgn}(\gamma) \pi
\end{gathered}
$$

This transformation causes the island O-points to become X-points, and vice versa, which is a natural consequence of a reversal in sign of the reconnected magnetic flux at the rational surface.

Note that our model neglects the influence of the perturbed bootstrap current, the perturbed ion polarization current, and magnetic field-line curvature, on island width evolution, on the assumption that these effects are much less important than the destabilizing effect of the resonant component of the RMP.

\section{G. Toroidal Ion Velocity Profile}

It is helpful to define the flux-surface label $k=[(1+\Omega) / 2]^{1 / 2}$. Thus, the island O-point corresponds to $k=0$, and the magnetic separatrix to $k=1$. Furthermore, $|X| \simeq 2 k$ in the limit $k \gg 1$. Let

$$
\hat{V}_{\varphi i}(k)=-\frac{V_{\varphi i}(k)}{\left(R_{0} / n_{\varphi}\right) \omega_{D}}
$$

where $V_{\varphi i}(k)$ is the toroidal ion velocity profile. (Note that the toroidal ion velocity-or, to be more exact, the parallel ion velocity - is a magnetic flux-surface function. ${ }^{27}$ ) According to the analysis of Ref. 27,

$$
\begin{aligned}
& \hat{V}_{\varphi i}(k<1)=\frac{d \phi_{p}}{d T} \\
& \hat{V}_{\varphi i}(k>1)=\frac{d \phi_{p}}{d T}+\frac{1}{(1+\bar{\nu}) \mathcal{A C} f}\left[\bar{\nu}\left(\gamma-\frac{d \phi_{p}}{d T}\right)-\frac{1}{I_{1}}\left(1-\frac{\bar{\nu}}{f}\right) \frac{d^{2} \phi_{p}}{d T^{2}}\right] .
\end{aligned}
$$

Here, $\bar{\nu}=\nu_{\perp i} / \nu_{\theta i}$, the function $f(k)$ is defined in Appendix $\mathrm{B}$, and the functions $\mathcal{A}(k)$ and $\mathcal{C}(k)$ are defined in Appendix $\mathrm{C}$. 


\section{H. Density and Temperature Profiles}

The electron number density profile in the vicinity of the island chain is a magnetic flux-surface function that takes the form

$$
\begin{aligned}
& n_{e}(k<1)=n_{0} \\
& n_{e}(k>1)=n_{0}\left[1-\operatorname{sgn}(X) \frac{w}{L_{n}} \int_{1}^{k} \frac{2 d k}{\mathcal{C}}\right] .
\end{aligned}
$$

The ion temperature profile in the vicinity of the island chain is a magnetic flux-surface function that takes the form

$$
\begin{aligned}
& T_{i}(k<1)=T_{i 0}, \\
& T_{i}(k>1)=T_{i 0}\left[1-\operatorname{sgn}(X) \frac{\eta_{i} w}{L_{n}} \int_{1}^{k} \frac{2 d k}{\mathcal{C}}\right] .
\end{aligned}
$$

Finally, the electron temperature profile in the vicinity of the island chain is a magnetic flux-surface function that takes the form

$$
\begin{aligned}
& T_{e}(k<1)=T_{e 0}, \\
& T_{e}(k>1)=T_{e 0}\left[1-\operatorname{sgn}(X) \frac{\eta_{e} w}{L_{n}} \int_{1}^{k} \frac{2 d k}{\mathcal{C}}\right] .
\end{aligned}
$$

Here, $T_{e 0}=T_{e}\left(r_{s}\right)$ and $\eta_{e}=\left(d \ln T_{e} / d \ln n_{e}\right)_{r=r_{s}}$, where $T_{e}(r)$ is the equilibrium electron temperature profile. Note that the density, ion temperature, and electron temperature, profiles are all flattened inside the island separatrix.

\section{APPROXIMATE ANALYTIC SOLUTIONS}

\section{A. Introduction}

Before attempting to solve Eqs. (7) and (8) numerically, it is helpful to search for approximate analytic solutions of these equations. 


\section{B. Renormalization}

The analytic solution of Eqs. (7) and (8) is facilitated by defining the following rescaled variables:

$$
\begin{aligned}
\hat{T} & =\gamma T, \\
\hat{\lambda}_{R} & =\gamma^{4 / 3} \lambda_{R}, \\
\hat{b}_{v} & =\frac{b_{v}}{\gamma^{2 / 3}}, \\
\hat{\xi} & =\frac{\xi}{\gamma} .
\end{aligned}
$$

When re-expressed in terms of these new variables, Eqs. (17) and (8) take the form

$$
\begin{aligned}
\hat{\lambda}_{R} \frac{d \hat{\xi}}{d \hat{T}} & =-\hat{\xi}^{2 / 3}+\hat{b}_{v} \cos \phi_{p}, \\
\gamma \frac{d^{2} \phi_{p}}{d \hat{T}^{2}}+\frac{d \phi_{p}}{d \hat{T}}+\hat{b}_{v} \hat{\xi}^{1 / 3} \sin \phi_{p} & =1 .
\end{aligned}
$$

Note that we are assuming, without loss of generality, that $\gamma>0$.

\section{Locked Regime}

Let us search for a locked solution of Eqs. (32) and (33) in which $\hat{\xi}$ and $\phi_{p}$ are both constant in time (i.e., $d / d \hat{T}=0$, which implies that the island chain is stationary in the laboratory frame). In this case, it is easily demonstrated that

$$
\begin{aligned}
\hat{\xi} & =\hat{b}_{v}^{3 / 2} \cos ^{3 / 2} \phi_{p}, \\
\hat{b}_{v}^{3 / 2} \cos ^{1 / 2} \phi_{p} \sin \phi_{p} & =1
\end{aligned}
$$

The previous two equations reveal that locked solutions exist as long as $\hat{b}_{v}>\hat{b}_{v}$ unlock, where

$$
\hat{b}_{v \text { unlock }}=\left(\frac{\sqrt{27}}{2}\right)^{1 / 3}=1.374 .
$$

Moreover, such solutions are characterized by $0 \leq \phi_{p} \leq \phi_{p \text { unlock}}$, where

$$
\phi_{p \text { unlock }}=\sin ^{-1}\left(\sqrt{\frac{2}{3}}\right)=54.7^{\circ} .
$$


Finally,

$$
\frac{w}{w_{v}}=\frac{\hat{\xi}^{1 / 3}}{\hat{b}_{v}^{1 / 2}}=\cos ^{1 / 2} \phi_{p} .
$$

Given that $\cos \phi_{p}>1 / \sqrt{3}=0.5774$ for locked solutions, we deduce that the locked island width, $w$, is similar in magnitude to the vacuum island width, $w_{v}$. In other words, there is no effective shielding in the so-called locked regime.

\section{Pulsating Regime}

Let us search for a solution of Eqs. (32) and (33) in which the island rotates in the laboratory frame (i.e., $d / d \hat{T} \neq 0$ ). Suppose that the term on the left-hand side of Eq. (32), and the first term on the left-hand side of Eq. (33), are both negligible. In this case, Eqs. (32) and (33) reduce to

$$
\begin{aligned}
\hat{\xi}^{2 / 3} & =\hat{b}_{v} \cos \phi_{p} \\
\frac{d \phi_{p}}{d \hat{T}}+\hat{b}_{v} \hat{\xi}^{1 / 3} \sin \phi_{p} & =1 .
\end{aligned}
$$

If the second term on the left-hand side of Eq. (40) is negligible then $d \phi_{p} / d \hat{T}=1$, which justifies the neglect of the first term on the left-hand side of Eq. (33). Equation (39) yields

$$
\hat{\xi}=\hat{b}_{v}^{3 / 2} \cos ^{3 / 2} \phi_{p}
$$

Obviously, this solution is only valid when $\cos \phi_{p} \geq 0$, which implies that $-\pi / 2 \leq \phi_{p} \leq \pi / 2$. Moreover,

$$
\frac{w}{w_{v}}=\left|\cos \phi_{p}\right|^{1 / 2}
$$

It follows that the island width pulsates, periodically falling to zero, at which times the island helical phase - which, otherwise, increases continually in time-jumps from $\pi / 2$ to $-\pi / 2{ }^{21,27,39}$ Furthermore, because the maximum allowed value of $\cos \phi_{p}$ is 1 , we deduce that there is no effective shielding in the so-called pulsating regime [i.e., $w / w_{v} \sim \mathcal{O}(1)$ ].

Let us assume that the neglect of the second term on the left-hand side of Eq. (40) is justified as long as it is not possible to find a locked solution of this equation with $\xi$ given by Eq. (41). In other words, the neglect is justified as long as

$$
\hat{b}_{v}^{3 / 2} \cos ^{1 / 2} \phi_{p} \sin \phi_{p}=1
$$




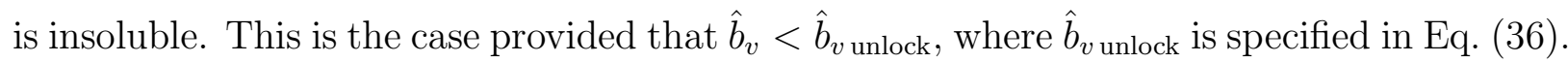

Finally, it is easily demonstrated that the neglect of the term on the left-hand side of Eq. (32) is justified provided

$$
\hat{b}_{v}<\frac{1}{\hat{\lambda}_{R}^{2}} .
$$

\section{E. Suppressed Regime}

Let us search for another rotating solution of Eqs. (32) and (33). Suppose that the first term on the right-hand side of Eq. (32), and the first term on the left-hand side of Eq. (33), are both negligible. In this case, Eqs. (32) and (33) reduce to

$$
\begin{aligned}
\hat{\lambda}_{R} \frac{d \hat{\xi}}{d \hat{T}} & =\hat{b}_{v} \cos \phi_{p} \\
\frac{d \phi_{p}}{d \hat{T}}+\hat{b}_{v} \hat{\xi}^{1 / 3} \sin \phi_{p} & =1 .
\end{aligned}
$$

If the second term on the left-hand side of Eq. (46) is negligible then $d \phi_{p} / d \hat{T}=1$, which justifies the neglect of the first term on the left-hand side of Eq. (333), and Eq. (45) can be integrated to give

$$
\hat{\xi}=\frac{\hat{b}_{v}}{\hat{\lambda}_{R}} \sin \phi_{p} .
$$

Obviously, this solution is only valid when $\sin \phi_{p} \geq 0$ (because $\hat{\xi}$ cannot be negative), which implies that $0 \leq \phi_{p} \leq \pi$. Moreover,

$$
\frac{w}{w_{v}}=\frac{\left|\sin \phi_{p}\right|^{1 / 3}}{\hat{b}_{v}^{1 / 6} \hat{\lambda}_{R}^{1 / 3}} .
$$

It follows that the island width pulsates, periodically falling to zero, at which times the island helical phase - which, otherwise, increases continually in time-jumps from $\pi$ to $0 . \underline{21,27}, 39$

Let us assume that the neglect of the second term on the left-hand side of Eq. (46) is justified as long as it is not possible to find a locked solution of this equation with $\xi$ given by Eq. (47). In other words, the neglect is justified as long as

$$
\frac{\hat{b}_{v}^{4 / 3}}{\hat{\lambda}_{R}^{1 / 3}} \sin ^{4 / 3} \phi_{p}=1 .
$$

is insoluble. This is the case provided that $\hat{b}_{v}<\hat{b}_{v \text { penetrate, where }}$

$$
\hat{b}_{v \text { penetrate }}=\hat{\lambda}_{R}^{1 / 4}
$$


Finally, it is easily demonstrated that the neglect of the first term on the right-hand side of Eq. (32) is justified provided

$$
\hat{b}_{v}>\frac{1}{\hat{\lambda}_{R}^{2}} .
$$

It follows, by comparison with Eq. (48), that the so-called suppressed regime is characterized by strong shielding (i.e., $w / w_{v} \ll 1$ ).

\section{F. Discussion}

The analysis in Sects. IIIC IIIE lead to the scenario illustrated schematically in Fig. 1, As shown in the figure, there are three solution regimes in $\hat{\lambda}_{R}-\hat{b}_{v}$ space. Namely, the locked, pulsating, and the suppressed regimes. Only the suppressed regime is characterized by strong shielding (i.e., $w / w_{v} \ll 1$ ). Thus, referring to the discussion in Para. 8 of Sect. 【, the shielded state corresponds to the suppressed regime, whereas the penetrated state corresponds to the union of the locked and pulsating regimes. Note that there is a region of parameter space, labelled S/L in the figure, in which the suppressed and locked solution branches co-exist. There is a bifurcation from the suppressed to the locked solution branch when the upper (in $\hat{b}_{v}$ ) boundary of this region is crossed. Likewise, there is a bifurcation from the locked to the suppressed solution branch when the lower boundary of the region is crossed. The former bifurcation is characterized by the sudden loss of strong shielding, whereas the latter is characterized by the sudden onset of strong shielding. Thus, again referring to the discussion in Para. 8 of Sect. I, the penetration threshold corresponds to $\hat{b}_{v}>\hat{b}_{v \text { penetrate }} \simeq \hat{\lambda}_{R}^{1 / 4}$, whereas the de-penetration threshold corresponds to $\hat{b}_{v}<\hat{b}_{v \text { de-penetrate }} \simeq 1$.

It is clear, from Fig. 1, that in order to get strong shielding at the rational surface (i.e., in order to be in the suppressed regime) it is necessary that $\hat{\lambda}_{R}>1$. Making use of Eqs. (10), (16), and (29), this criterion reduces to $\omega_{0}>\omega_{0 \text { min }}$, where

$$
\omega_{0 \min } \tau_{H}=\frac{1}{S^{3 / 4}\left(\omega_{D} \tau_{H}\right)^{1 / 4}}
$$

It follows that there is a minimum level of plasma flow at the rational surface-parameterized by the natural frequency, $\omega_{0}$-required for strong shielding to be possible.

The maximum amount of shielding in the suppressed regime is achieved at the upper boundary of this regime in $\hat{\lambda}_{R}-\hat{b}_{v}$ space, which corresponds to $\hat{b}_{v} \simeq \hat{\lambda}_{R}^{1 / 4}$. It follows from 
Eqs. (10), (16), (29), (48), and (52) that

$$
\left(\frac{w}{w_{v}}\right)_{\min } \simeq \frac{1}{S^{3 / 8}\left(\omega_{0} \tau_{H}\right)^{1 / 2}\left(\omega_{D} \tau_{H}\right)^{1 / 8}}=\left(\frac{\omega_{0 \min }}{\omega_{0}}\right)^{1 / 2} .
$$

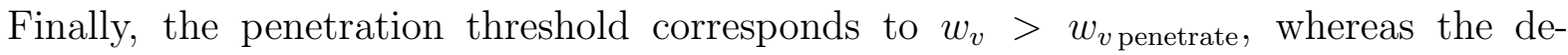
penetration threshold corresponds to $w_{v}<w_{v}$ de-penetrate, where

$$
\begin{gathered}
\frac{w_{v \text { penetrate }}}{r_{s}} \simeq S^{1 / 8}\left(\omega_{0} \tau_{H}\right)^{1 / 2}\left(\omega_{D} \tau_{H}\right)^{3 / 8}=\left(\frac{\omega_{0}}{\omega_{0 \min }}\right)^{1 / 2}\left(\frac{\omega_{D} \tau_{H}}{S}\right)^{1 / 4} \\
\frac{w_{v \text { de-penetrate }}}{r_{s}} \simeq\left(\omega_{0} \tau_{H}\right)^{1 / 3}\left(\omega_{D} \tau_{H}\right)^{1 / 3}=\left(\frac{\omega_{0}}{\omega_{0 \min }}\right)^{1 / 3}\left(\frac{\omega_{D} \tau_{H}}{S}\right)^{1 / 4}
\end{gathered}
$$

Here, use has been made of Eqs. (14) and (15).

\section{NUMERICAL SOLUTIONS}

\section{A. Introduction}

Let us now consider some example numerical solutions of Eqs. (7) and (8) .

\section{B. First Example}

Our first example is characterized by $\lambda_{R}=0.1, \gamma=1.0$, and $\bar{\nu}=0.1$. This is a case in which the island natural frequency is not large enough to enable strong shielding. (See Sect. IIIF.) The normalized resonant component of the RMP is increased linearly from a small value at $T=0.0$ to $b_{v}=2.0$ at $T=100.0$, and then decreased linearly to a small value at $T=200.0$. Referring to Fig. 1, we would expect to start off in the pulsating regime, to make a transition to the locked regime when $b_{v}$ exceeds a critical value similar to unity, and then to make a back transition to the pulsating regime when $b_{v}$ falls below the same critical value. It can be seen, from Fig. 2, that this is essentially what happens. The pulsating regime can be identified because the helical phase of the island is restricted to the range $-\pi / 2 \leq \phi_{p} \leq \pi / 2$, the island width periodically falls to zero, and there is no shielding [i.e., $\left.w / w_{v} \sim \mathcal{O}(1)\right]$. The locked regime can be identified because $\phi_{p}$ is relatively static, the island width has a relatively constant nonzero value, $\hat{V}_{\phi i}(k=0)=0$ (i.e., the toroidal flow velocity inside the island separatrix is reduced to zero), and there is no shielding. Note that, 
in the pulsating regime, the electromagnetic torque exerted by the resonant component of the RMP is strongly modulated, which gives rise to a modulation of the local toroidal ion velocity. On the other hand, in the locked regime, the torque is constant, and there is no modulation of the ion velocity. Incidentally, the modulation of the torque in the pulsating regime is an intrinsically nonlinear effect (i.e., a linear response model would give a constant torque). The actual transition from the pulsating to the locked regime takes place when $b_{v} \simeq 1.6$, whereas the back transition takes place when $b_{v} \simeq 1.2$. This is not quite what analysis presented in Sect. [II predicts, which is hardly surprising, given the approximate nature of the analysis.

Figure 3 shows simulated "Mirnov" data associated with the first example. The figure actually shows contours of $b_{r}=w^{2} \cos \left(\chi-\phi_{p}\right)$ plotted in $T-\chi$ space (recall that $\chi=$ $m_{\theta} \theta-n_{\varphi} \varphi$ ), and is meant to mimic the data that would be obtained from a comprehensive array of magnetic pick-up coils surrounding the plasma, such as was recently installed on the DIII-D tokamak. $\stackrel{40}{*}$ The pulsating regime appears as an interlocking pattern of small regions of positive and negative $b_{r}$ that are aligned almost almost parallel to the $\chi$ axis, but do not extend over all values of $\chi$. The locked regime appears as alternating thick bands of positive and negative $b_{r}$ that are aligned almost parallel to the $T$ axis.

\section{Second Example}

Our second example is characterized by $\lambda_{R}=10.0, \gamma=1.0$, and $\bar{\nu}=0.1$. This is a case in which the island natural frequency is large enough to enable moderate shielding. (See Sect. IIIF) The normalized resonant component of the RMP is increased linearly from a small value at $T=0.0$ to $b_{v}=2.0$ at $T=100.0$, and then decreased linearly to a small value at $T=200.0$. Referring to Fig. 1, we would expect to start off in the suppressed regime, to make a transition to the locked regime when $b_{v}$ exceeds a critical value somewhat larger than unity, and then to make a back transition to the suppressed regime when $b_{v}$ falls below a second critical value that is similar to unity. It can be seen, from Fig. 4, that this is essentially what happens. The suppressed regime can be identified because the helical phase of the island is restricted to the range $0 \leq \phi_{p} \leq \pi$, the island width periodically falls to zero, and there is moderate shielding (i.e., $w / w_{v} \leq 0.6$ ). As before, the locked regime can be identified because $\phi_{p}$ is relatively static, the island width has a 
relatively constant nonzero value, $\hat{V}_{\phi i}(k=0)=0$, and there is no shielding. Note that, in the suppressed regime, the electromagnetic torque exerted by the resonant component of the RMP is strongly modulated, which gives rise to a modulation of the local toroidal ion velocity. As before, the modulation of the torque in the suppressed regime is an intrinsically nonlinear effect (i.e., a linear response model would give a constant torque). Note, finally, that the driven island chain makes a full rotation during the back transition from the locked to the suppressed regimes; this is a vestigial version of the spin up described Sect. IVD,

Figure 5 shows simulated Mirnov data associated with the second example. The suppressed regime appears as an interlocking pattern of small regions of positive and negative $b_{r}$ that are aligned almost parallel to the $\chi$ axis, but do not extend over all values of $\chi$. As before, the locked regime appears as alternating thick bands of positive and negative $b_{r}$ that are aligned almost parallel to the $T$ axis.

\section{Third Example}

Our third example is characterized by $\lambda_{R}=100.0, \gamma=1.0$, and $\bar{\nu}=0.1$. This is a case in which the island natural frequency is large enough to enable strong shielding. (See Sect. IIIF.) The normalized resonant component of the RMP is increased linearly from a small value at $T=0.0$ to $b_{v}=3.0$ at $T=80.0$, decreased linearly to a small value at $T=160.0$, and, thereafter, held steady. Referring to Fig. 1, we would expect to start off in the suppressed regime, to make a transition to the locked regime when $b_{v}$ exceeds a critical value that is considerably larger than unity, and then to make a back transition to the suppressed regime when $b_{v}$ falls below a second critical value that is similar to unity. It can be seen, from Fig. 6, that this is essentially what happens, with one caveat (involving the spin up). The suppressed regime can be identified because the helical phase of the island is restricted to the range $0 \leq \phi_{p} \leq \pi$, the island width periodically falls to zero, and there is strong shielding (i.e., $w / w_{v} \leq 0.25$ ). As before, the locked regime can be identified because $\phi_{p}$ is relatively static, the island width has a relatively constant nonzero value, $\hat{V}_{\phi i}(k=0)=0$, and there is no shielding. Note, however, that at the end of the locked phase, instead of immediately re-entering the suppressed regime, the island chain spins up: i.e., its helical phase increases continually in time. This behavior occurs because the island chain cannot decay away fast enough to prevent it from being entrained by the re-accelerated 
plasma flow at the rational surface. Of course, the island chain will eventually re-enter the suppressed regime, but only when enough time has elapsed for its width to decay to zero: i.e., after 100 , or so, normalized time units.

Figure 7 shows simulated Mirnov data associated with the third example. As before, the suppressed regime appears as an interlocking pattern of small regions of positive and negative $b_{r}$ that are aligned almost parallel to the $\chi$ axis, but do not extend over all values of $\chi$. However, in this particular example, it is much easier to see that the positive and negative regions of the suppressed regime predominately occupy the same ranges of $\chi$ as the positive and negative bands in the locked regime. As before, the locked regime appears as alternating thick bands of positive and negative $b_{r}$ that are aligned almost parallel to the $T$ axis. Finally, the spin up appears as alternating diagonal bands of positive and negative $b_{r}$ that extend over all values of $\chi$.

\section{E. Fourth Example}

Our fourth example is characterized by $\lambda_{R}=100.0, b_{v}=0.6$, and $\bar{\nu}=0.1$. This is a case in which the island natural frequency is initially large enough to enable strong shielding. (See Sect. [IIF.) The normalized island natural frequency is ramped linearly from $\gamma=1.0$ at $T=0.0$ to $\gamma=-1.0$ at $T=200.0$. This particular example is designed to illustrate what happens when the natural frequency at the rational surface passes through zero. As can be seen from Figs. 8 and 9, we start off in the suppressed regime, there is a transition to the locked regime when $\gamma$ becomes sufficiently small, and then when $|\gamma|$ becomes sufficiently large the locked island chain spins up and decays aways. The only major difference between this example and the previous one is that the island spins up to a negative rotation frequency, because $\gamma$ has become negative by the time the locked island chain unlocks. Note, finally, that the locked phase is centered on the time at which the normalized natural frequency, $\gamma$, passes through zero. In other words, the breakdown of strong shielding is clearly associated with island natural frequency passing through zero. 


\section{SUMMARY AND DISCUSSION}

The aim of this paper is to develop a physical understanding of the dynamics of magnetic reconnection driven at a single, tearing-stable, rational surface that is resonant with one of the dominant helical harmonics of an applied RMP. The rational surface is assumed to be located just outside, but close to the top of, the pedestal of an H-mode tokamak plasma. Over the years, many different theoretical approaches have been taken to investigate driven reconnection at an intrinsically stable rational surface embedded in a tokamak plasma. These include single-fluid linear response models, two-fluid linear response models, kinetic linear response models, single-fluid nonlinear response models, and two-fluid nonlinear response models. However, it is the thesis of this paper that the correct response model is a two-fluid nonlinear model, and that all of the other models mentioned previously are either inadequate or invalid. (See the discussion in Sect. I.)

The two-fluid nonlinear response model discussed in this paper consists of an island width evolution equation and an island phase evolution equation. (See Sect. IIF.) These two equations are coupled together. The island width and phase evolution equations are sufficiently nonlinear that they can only be solved accurately by numerical means. However, it is possible to find approximate analytical solutions of these equations. (See Sect. III.) These analytic solutions reveal that there are three different response regimes - namely, the locked, pulsating, and suppressed regimes. In the locked regime, the magnetic island chain driven at the rational surface has a constant phase relative to the resonant component of the RMP: i.e., the island chain is stationary in the laboratory frame. Moreover, the width of the island chain is similar to the vacuum island width, which implies that there is no effective "shielding" (i.e., suppression of driven magnetic reconnection) in this regime. In the pulsating and suppressed regimes, the driven island chain is forced to rotate by plasma flow at the rational surface. However, the island width periodically falls to zero in both regimes, at which times the helical phase of the island chain jumps by $\pi$ radians. In both regimes, the electromagnetic torque exerted by the resonant component of the RMP is strongly modulated, which gives rise to a modulation of the local toroidal ion velocity. The main difference between the pulsating and the suppressed regimes is that there is no effective shielding in the former regime (i.e., the driven island width is similar to the vacuum island width), whereas there is strong shielding in the latter regime (i.e., the driven island width 
is much smaller than the vacuum island width). There exists a region of parameter space in which the suppressed and the locked solution branches co-exist. Bifurcations from one solution branch to the other are triggered when the boundaries of this region are crossed. These bifurcations are characterized by the sudden loss of strong shielding, or the sudden onset of strong shielding.

Numerical integration of the island width and island phase evolution equations yields results that are consistent with the aforementioned approximate analytic solutions, with one proviso. (See Sect. IV]) Namely, that the transition from the locked regime to the suppressed regime is characterized by an intermediate regime in which the island chain spins up: i.e., its helical phase increases continually in time. This behavior occurs because the island chain cannot decay away fast enough to prevent it from being entrained by the re-accelerated plasma flow at the rational surface.

\section{ACKNOWLEDGEMENTS}

This research was funded by the U.S. Department of Energy under contract DE-FG0204ER-54742.

1 F. Wagner, G. Becker, K. Behringer, D. Campbell, A. Eberhagen, W. Engelhardt, et al., Phys. Rev. Lett. 49, 1408 (1982).

2 H. Zohm, Plasma Phys. Control. Fusion 38, 105 (1996).

3 A. Loarte, G. Saibene, R. Sartori, M. Bécoulet, L. Horton, T. Eich, et al., J. Nucl. Materials 313-316, 962 (2003).

4 T.E. Evans, R.A. Moyer, P.R. Thomas, J.G. Watkins, T.H. Osborne, J.A. Boedo, et al., Phys. Rev. Lett. 92, 235003 (2004).

5 Y. Liang, H.R. Koslowski, P.R. Thomas, E. Nardon, B. Alper, P. Andrew, et al., Phys. Rev. Lett. 98, 265004 (2007).

6 W. Suttrop, T. Eich, J.C. Fuchs, S. Günter, A. Janzer, A. Herrmann, et al., Phys. Rev. Lett. 106, 225004 (2011). 
7 Y.M. Jeon, J.-K. Park, S.W. Yoon, W.H. Ko, S.G. Lee, K.D. Lee, et al., Phys. Rev. Lett. 109, 035004 (2012).

8 Y. Sun, Y. Liang, Y.Q. Liu, S. Gu, X. Yang, W. Guo, et al., Phys. Rev. Lett. 117, 115001 (2016).

9 T.E. Evans, M.E. Fenstermacher, R.A. Moyer, T.H. Osborne, J.G. Watkins, P. Gohil, et al., Nucl. Fusion 48, 024002 (2008).

10 C.C. Petty, T.E. Evans, J.C. DeBoo, B. Hudson, R.J. La Haye, T.C. Luce, et al., Nucl. Fusion 50, $022002(2010)$.

11 R.A. Moyer, C. Paz-Soldan, R. Nazikian, D.M. Orlov, N.M. Ferraro, B.A. Grierson, et al., Phys. Plasmas 24, 102501 (2017).

12 ITER Physics Basis Editors, ITER Physics Expert Group Chairs and Co-Chairs and ITER Joint Central Team and Physics Integration Unit, Nucl. Fusion 12, 2137 (1999).

13 J.W. Connor, R.J. Hastie, H.R. Wilson, and R.L. Miller, Phys. Plasmas 5, 2687 (1998).

14 M.E. Fenstermacher, T.E. Evans, T.H. Osborne, M.J. Schaffer, M.P. Aldan, J.S. deGrassie, et al., Phys. Plasmas 15, 056122 (2008).

15 M. Bécoulet, F. Orain, P. Maget, N. Mellet, X. Garbet, E. Nardon, et al., Nucl. Fusion 52, $054003(2012)$.

16 P.B. Snyder, R.J. Groebner, J.W. Hughes, T.H. Osborne, M. Beurskens, A.W. Leonard, H.R. Wilson, and X.Q. Xu, Nucl. Fusion 51, 103016 (2011).

17 P.B. Snyder, T.H. Osborne, K.H. Burrell, R.J. Groebner, A.W. Leonard, R. Nazikian, et al., Phys. Plasmas 19, 056115 (2012).

18 M.R. Wade, R. Nazikian, J.S. deGrassie, T.E. Evans, N.M. Ferraro, R.A. Moyer, et al., Nucl. Fusion 55, 023002 (2015).

19 Z. Chang, and J.D. Callen, Nucl. Fusion 30, 219 (1990).

20 R. Fitzpatrick, Nucl. Fusion 33, 1049 (1993).

21 R. Fitzpatrick, Phys. Plasmas 5, 3325 (1998).

22 Y.Q. Liu, J.W. Connor, S.C. Cowley, C.J. Ham, R.J. Hastie, and T.C. Hender, Phys. Plasmas 19, 072509 (2012). 
23 F.L. Waelbroeck, Phys. Plasmas 10, 4040 (2003).

24 A. Cole, and R. Fitzpatrick, Phys. Plasmas 13, 032503 (2006).

25 F.L. Waelbroeck, I. Joseph, E. Nardon, M. Bécoulet, and R. Fitzpatrick, Nucl. Fusion 52, $074004(2012)$.

26 M.F. Heyn, I.B. Ivanov, S.V. Kasilov, W. Kernbichler, I. Joseph, R.A. Moyer, and A.M. Runov, Nucl. Fusion 48, 024005 (2008).

27 R. Fitzpatrick, Nonlinear Neoclassical Two-Fluid Theory of Response of Tokamak Plasma to Resonant Error-Field, to appear in Physics of Plasmas (2018).

28 J.D. Callen, R. Nazikian, C. Paz-Soldan, N.M. Ferraro, M.T. Beidler, C.C. Hegna, and R.J. LaHaye, Model of $n=2$ RMP ELM Suppression in DIII-D, UW-CPTC 16-4 (2016). https://cptc.wiscweb.wisc.edu/wp-content/uploads/sites/327/2017/09/UW-CPTC_16-4.pdf

29 R. Nazikian, C. Paz-Soldan, J.D. Callen, J.S. deGrassie, D. Eldon, T.E. Evans, et al., Phys. Rev. Lett. 114, 105002 (2015).

30 R. Nazikian, C.C. Petty, A. Bortolon, X. Chen, D. Eldon, T.E. Evans, et al., Nucl. Fusion 58, 106010 (2018).

31 R.D. Hazeltine, M. Kotscheneuther, and P.J. Morrison, Phys. Fluids 28, 2466 (1985).

32 H.P. Furth, J. Killeen, and M.N. Rosenbluth, Phys. Fluids 6, 459 (1963).

33 R. Fitzpatrick, P.G. Watson, and F.L. Waelbroeck, Phys. Plasmas 12, 082510 (2005).

34 R. Fitzpatrick, Phys. Plasmas 23, 052506 (2016).

35 R.J. La Haye, C.C. Petty, E.J. Strait, F.L. Waelbroeck, and H.R. Wilson, Phys. Plasmas 10, 3644 (2003).

36 P. Buratti, E. Alessi, M. Baruzzo, A. Casolari, E. Giovannozzi, C. Giroud, N. Hawkes, S. Menmuir, G. Purcella, and J. Contributors, Nucl. Fusion 56, 076004 (2016).

37 G. Ara, B. Basu, B. Coppi, G. Laval, M.N. Rosenbluth, and B.V. Waddell, Ann. Phys. (NY) 112, 443 (1978).

38 Y.B. Kim, P.H. Diamond, and R.J. Groebner, Phys. Fluids B 3, 2050 (1991).

39 R. Fitzpatrick, Phys. Plasmas 21, 092513 (2014). 
40 J.D. King, E.J. Strait, R.L. Boivin, D. Taussig, M.G. Watkins, J.M. Hansen, et al., Rev. Sci. Instrum. 85, 083503 (2014).

41 J.A. Wesson, Tokamaks, 3rd Edition. (Oxford University Press, 2004).

42 K.C. Shaing, S.A. Sabbagh, and M.S. Chu, Nucl. Fusion 50, 025022 (2010).

\section{Appendix A: Neoclassical Velocity Coefficients}

According to Ref. 38, we can write

$$
\lambda_{\theta i}=\frac{5}{2}-\frac{1 / \sqrt{2}+\alpha}{\sqrt{2}-\ln (1+\sqrt{2})+\alpha},
$$

where $\alpha=Z_{\text {eff }}-1$. Here, $Z_{\text {eff }}$ is the conventional measure of plasma impurity content $\underline{41}$ Furthermore, it is assumed that the majority ions have charge number unity, and are in the banana collisionality regime.

According to Ref. 42, we can write

$$
\lambda_{\perp i}=\int_{0}^{\infty} \frac{x^{9} \mathrm{e}^{-x^{2}}\left(x^{2}-5 / 2\right) d x}{F(x)+\alpha} / \int_{0}^{\infty} \frac{x^{9} \mathrm{e}^{-x^{2}} d x}{F(x)+\alpha},
$$

where $F(x)=\Phi(x)-G(x), G(x)=\left[\Phi(x)-x \Phi^{\prime}(x)\right] /\left(2 x^{2}\right), \Phi(x)$ is a standard error function, and ' denotes a derivative with respect to argument. Here, it is assumed that the majority ions have charge number unity, and are in the $1 / \nu$ collisionality regime.

Table I illustrates the dependence of the neoclassical velocity parameters, $\lambda_{\theta i}$ and $\lambda_{\perp i}$, on $Z_{\text {eff }}$.

\section{Appendix B: Useful Integrals}

Let

$$
\begin{aligned}
I_{i} & =\int_{0}^{\infty} \frac{64\left[\left(k^{2}-1 / 2\right) \mathcal{A}-k^{2} \mathcal{C}\right]^{2}}{\mathcal{A}} d k \\
I_{v} & =\left(\frac{\bar{\nu}}{1+\bar{\nu}}\right) \int_{1}^{\infty} \frac{8(\mathcal{A C}-1)}{\mathcal{A} \mathcal{C}^{2} f} d k \\
I_{1} & =\frac{1}{I_{v}} \int_{1}^{\infty} \frac{8(\mathcal{A C}-1)^{2}}{\mathcal{A C} \mathcal{C}^{2} f^{2}} d k
\end{aligned}
$$

where $\bar{\nu}=\nu_{\perp i} / \nu_{\theta i}$, and

$$
f(k)=1-\frac{1}{1+\bar{\nu}} \frac{1}{\mathcal{A C}}
$$




\begin{tabular}{llll}
\hline$Z_{\text {eff }}$ & $\lambda_{\theta i}$ & $\lambda_{\perp i}$ & $\eta_{i \text { crit }}$ \\
\hline 1.0 & 1.173 & 2.367 & 5.78 \\
2.0 & 1.386 & 2.440 & 2.59 \\
3.0 & 1.431 & 2.461 & 2.32 \\
4.0 & 1.451 & 2.471 & 2.22 \\
5.0 & 1.462 & 2.477 & 2.17 \\
$\infty$ & 1.5 & 2.5 & 2.0 \\
\hline
\end{tabular}

TABLE I. Neoclassical velocity coefficients as functions of $Z_{\text {eff }} \cdot \eta_{i \text { crit }}=1 /\left(\eta_{\theta i}-1\right)$ is the critical value of $\eta_{i}$ at the rational surface above which the natural frequency is offset from the local $\mathbf{E} \times \mathbf{B}$ frequency in the electron diamagnetic direction, as opposed to the ion diamagnetic direction.

Here, the functions $\mathcal{A}(k)$ and $\mathcal{C}(k)$ are defined in Appendix $\mathrm{C}$.

It is easily demonstrated that $\underline{27}$

$$
\begin{aligned}
I_{i} & =3.2908, \\
I_{v} & =\left\{\begin{array}{ll}
2^{1 / 4} \pi \bar{\nu}^{3 / 4} & \bar{\nu} \ll 1 \\
0.35724 & \bar{\nu} \gg 1
\end{array},\right. \\
I_{1} & =\left\{\begin{array}{ll}
0.75 / \bar{\nu} & \bar{\nu} \ll 1 \\
0.18182 & \bar{\nu} \gg 1
\end{array} .\right.
\end{aligned}
$$

\section{Appendix C: Useful Functions}

$$
\begin{aligned}
& \mathcal{A}(k<1)=\left(\frac{2}{\pi}\right) k K(k) \\
& \mathcal{A}(k>1)=\left(\frac{2}{\pi}\right) K\left(\frac{1}{k}\right) \\
& \mathcal{C}(k<1)=\left(\frac{2}{\pi}\right) \frac{\left[E(k)+\left(k^{2}-1\right) K(k)\right]}{k}, \\
& \mathcal{C}(k>1)=\left(\frac{2}{\pi}\right) E\left(\frac{1}{k}\right) .
\end{aligned}
$$


Here,

$$
\begin{aligned}
& E(x)=\int_{0}^{\pi / 2}\left(1-x^{2} \sin ^{2} u\right)^{1 / 2} d u \\
& K(x)=\int_{0}^{\pi / 2}\left(1-x^{2} \sin ^{2} u\right)^{-1 / 2} d u
\end{aligned}
$$

are standard complete elliptic integrals. 


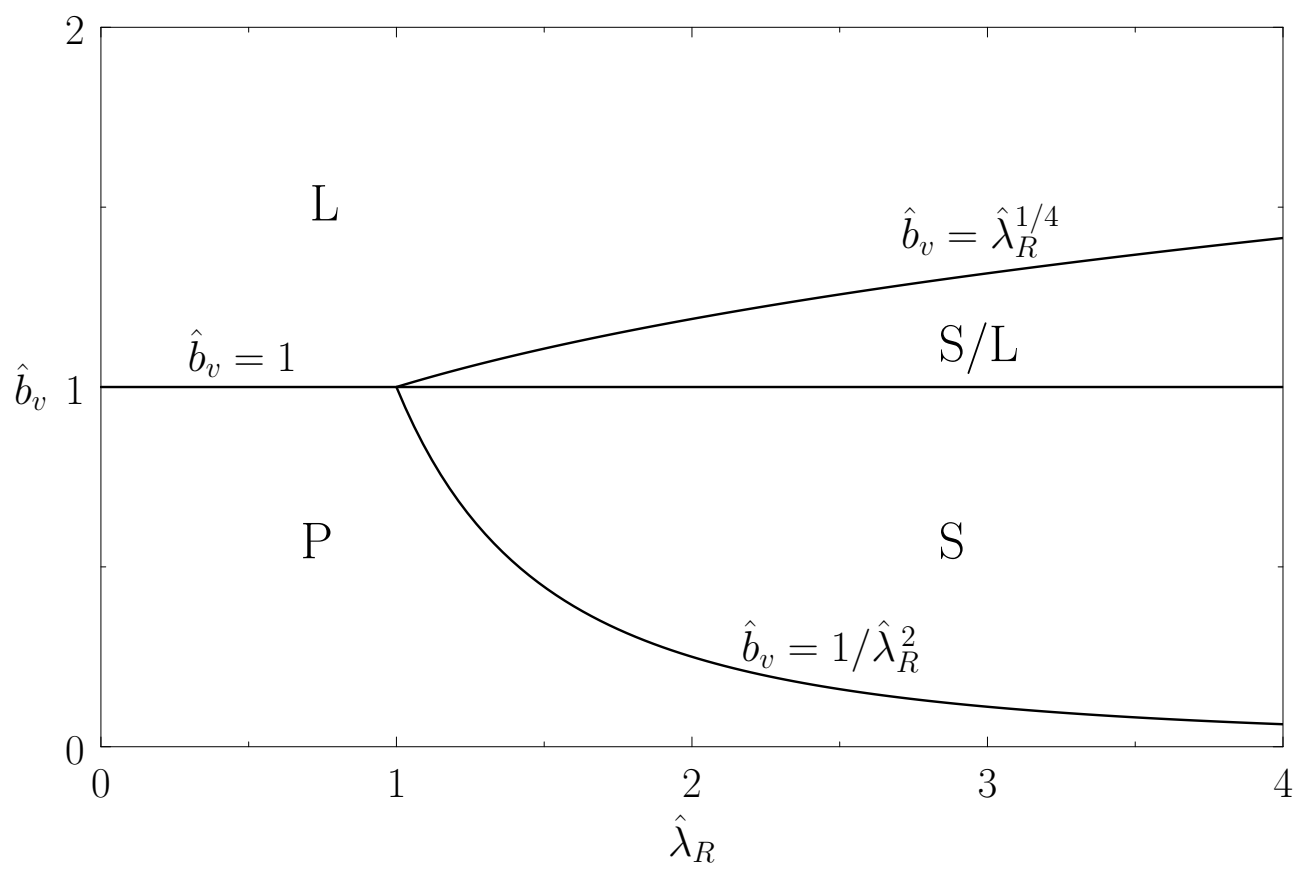

FIG. 1. Solution regimes for a two-fluid nonlinear response model of driven magnetic reconnection at a single, tearing-stable rational surface, embedded in a tokamak plasma, plotted in $\hat{\lambda}_{R}-\hat{b}_{v}$ space. $\mathrm{L}, \mathrm{P}$, and $\mathrm{S}$ refer to the locked regime, the pulsating regime, and the suppressed regime, respectively. 

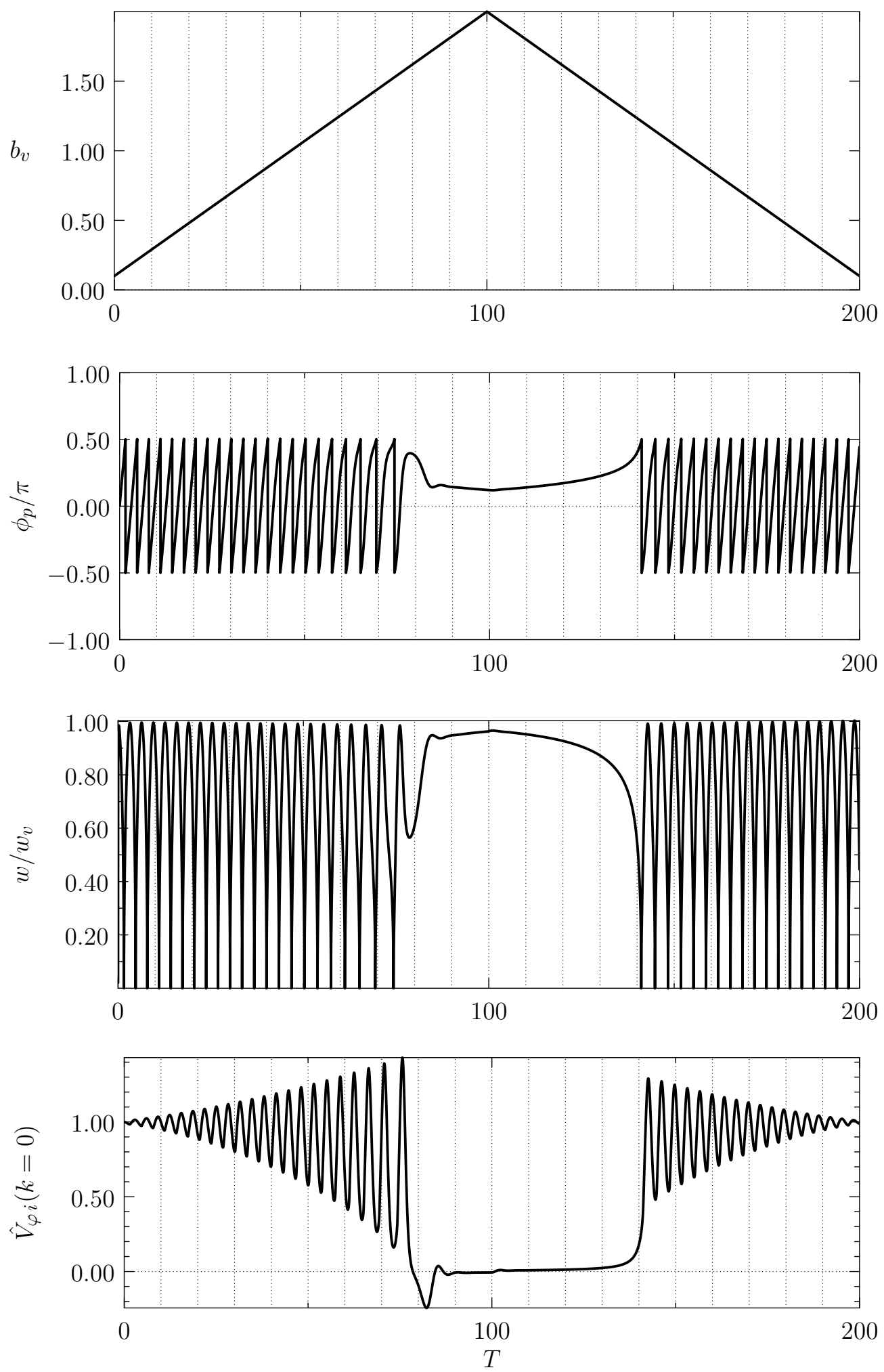

FIG. 2. Numerical solution of Eqs. (77) and (8) with $\lambda_{R}=0.1, \gamma=1.0$, and $\bar{\nu}=0.1$. In order from the top to the bottom, the panels show the normalized resonant component of the RMP, the island helical phase, the ratio of the island width to the vacuum island width, and the normalized toroidal ion velocity inside the island separatrix. 

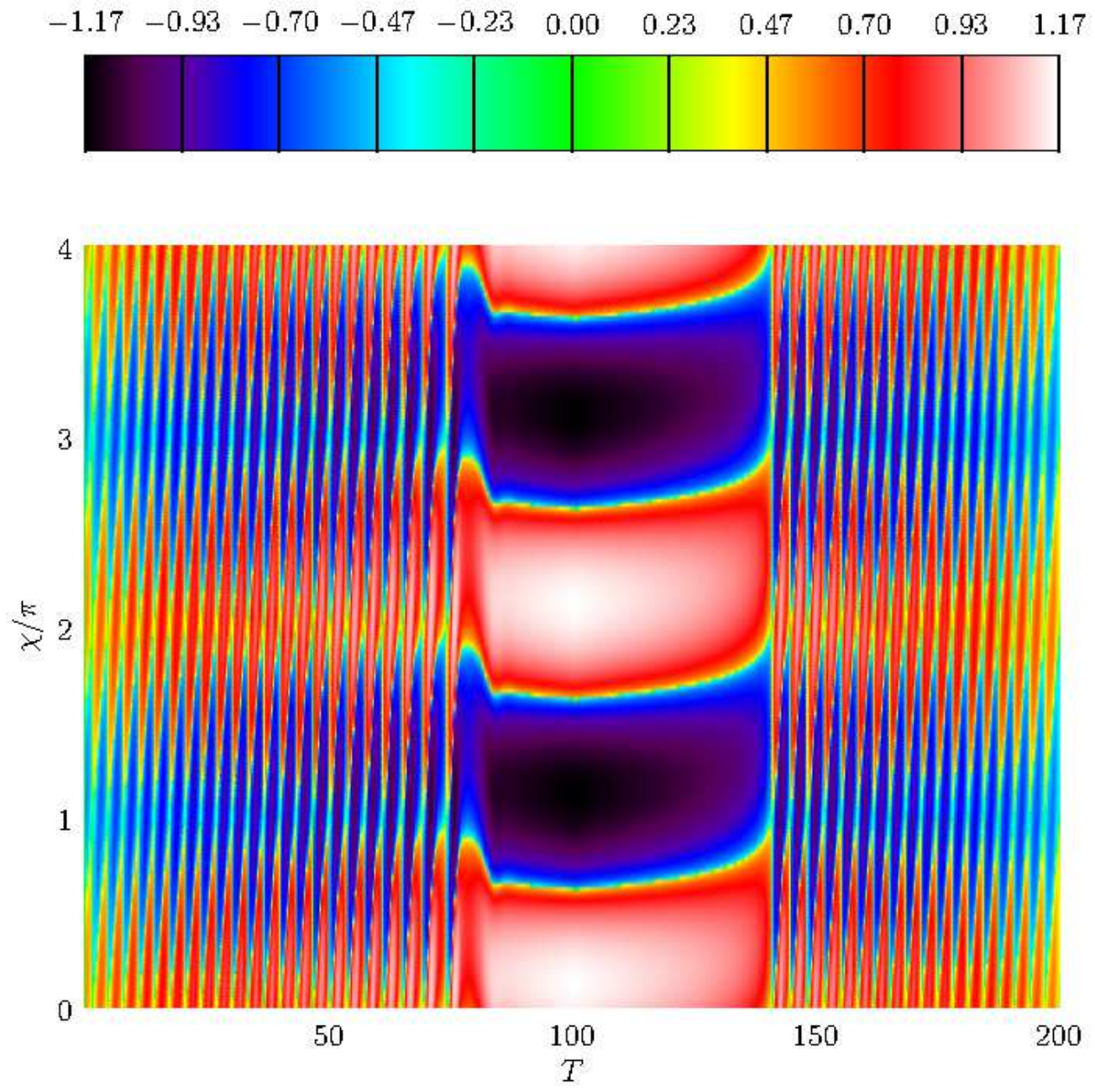

FIG. 3. Simulated Mirnov data for the case shown in Fig. 2. 

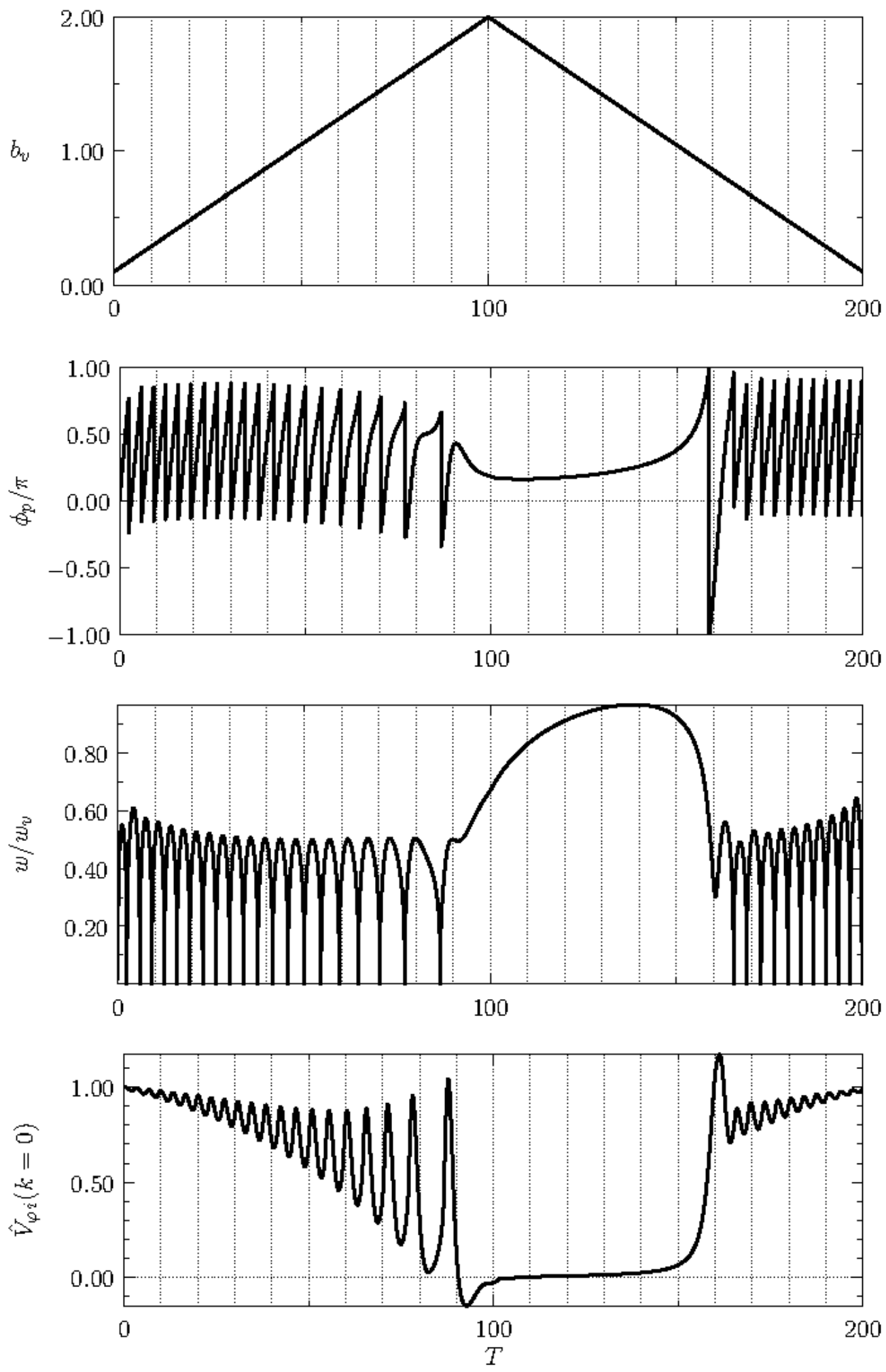

FIG. 4. Numerical solution of Eqs. (77) and (8) with $\lambda_{R}=10.0, \gamma=1.0$, and $\bar{\nu}=0.1$. In order from the top to the bottom, the panels show the normalized resonant component of the RMP, the island helical phase, the ratio of the island width to the vacuum island width, and the normalized toroidal ion velocity inside the island separatrix. 

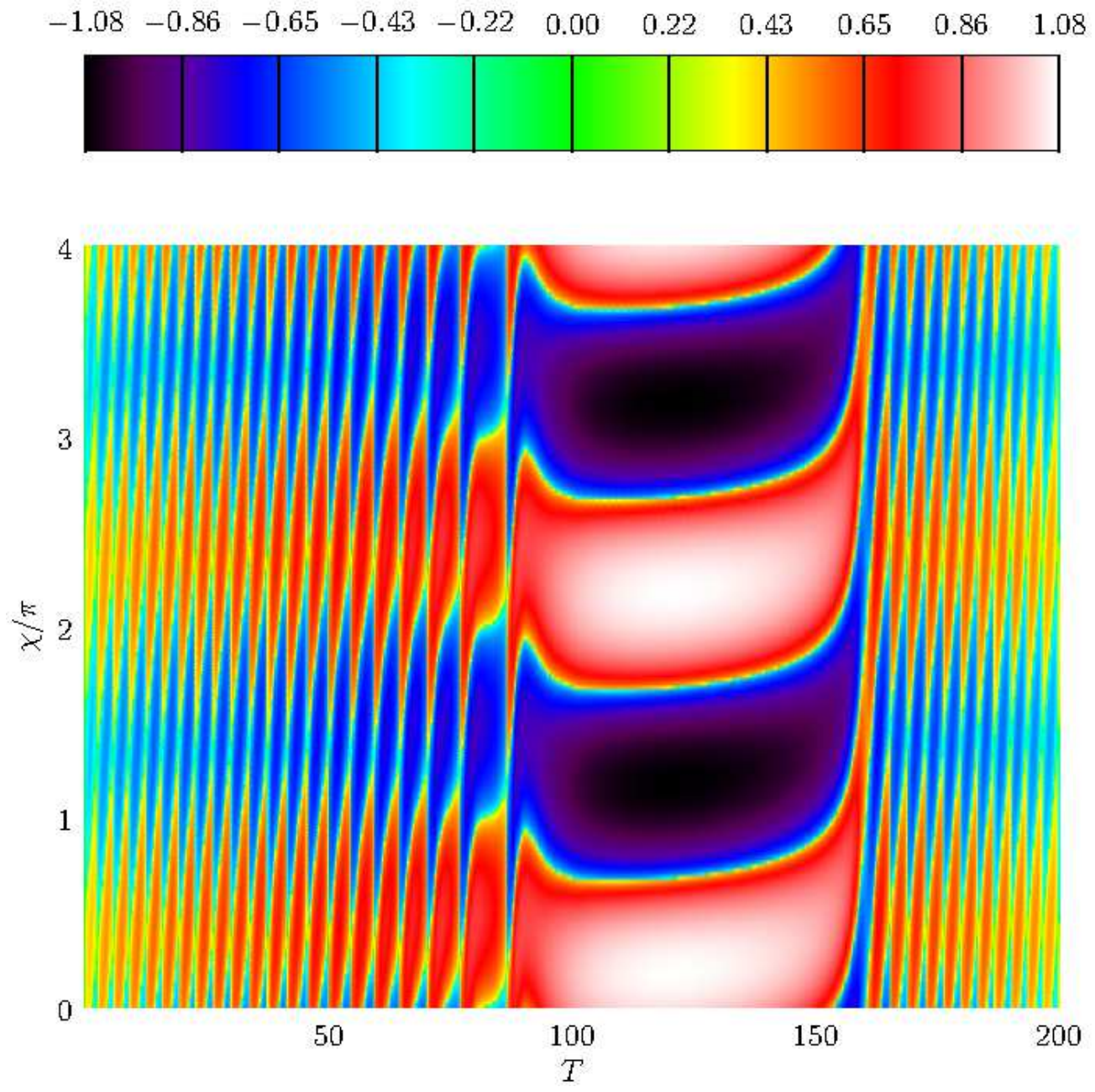

FIG. 5. Simulated Mirnov data for the case shown in Fig. 4. 

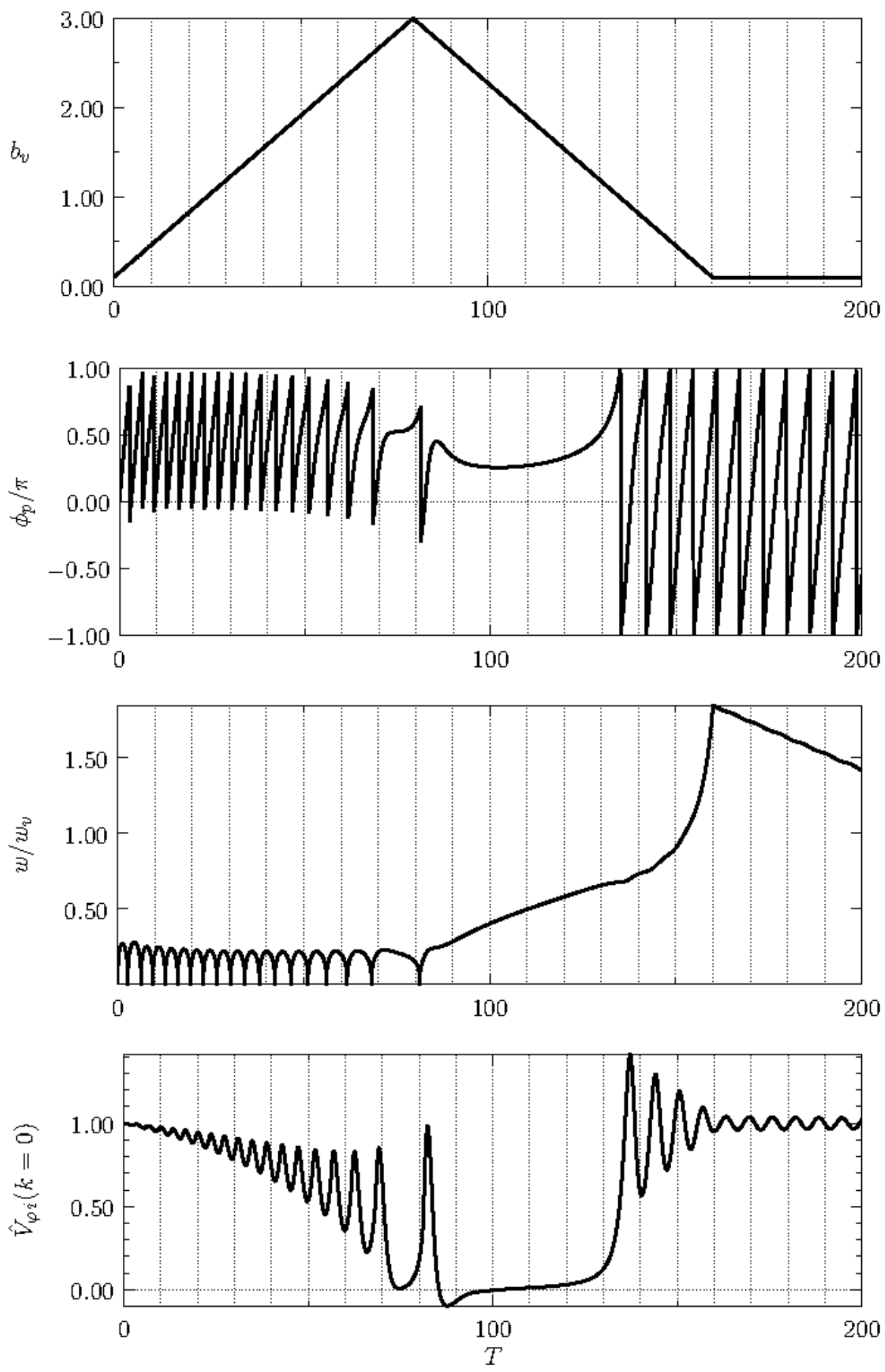

FIG. 6. Numerical solution of Eqs. (7) and (8) with $\lambda_{R}=100.0, \gamma=1.0$, and $\bar{\nu}=0.1$. In order from the top to the bottom, the panels show the normalized resonant component of the RMP, the island helical phase, the ratio of the island width to the vacuum island width, and the normalized toroidal ion velocity inside the island separatrix. 

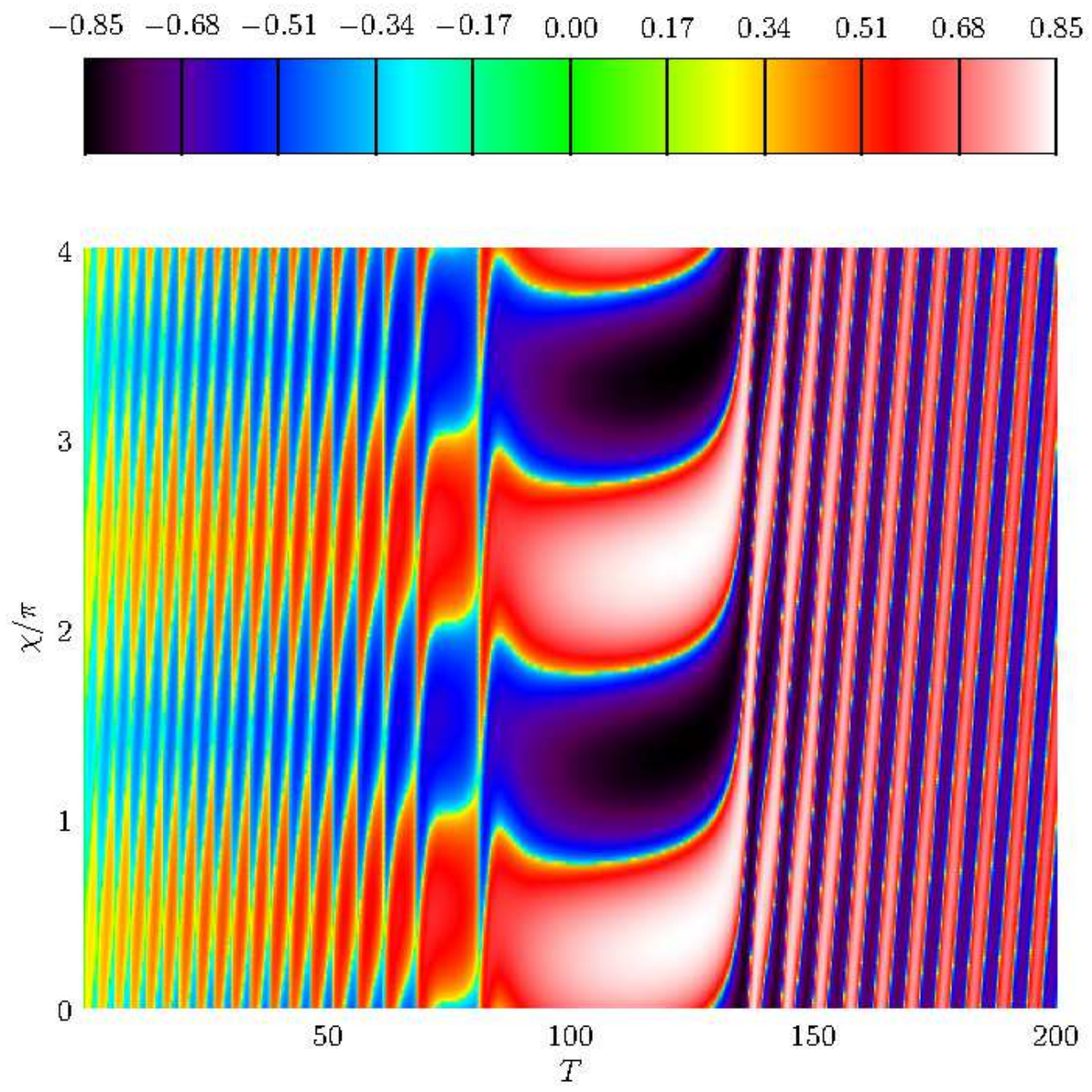

FIG. 7. Simulated Mirnov data for the case shown in Fig. 6. 

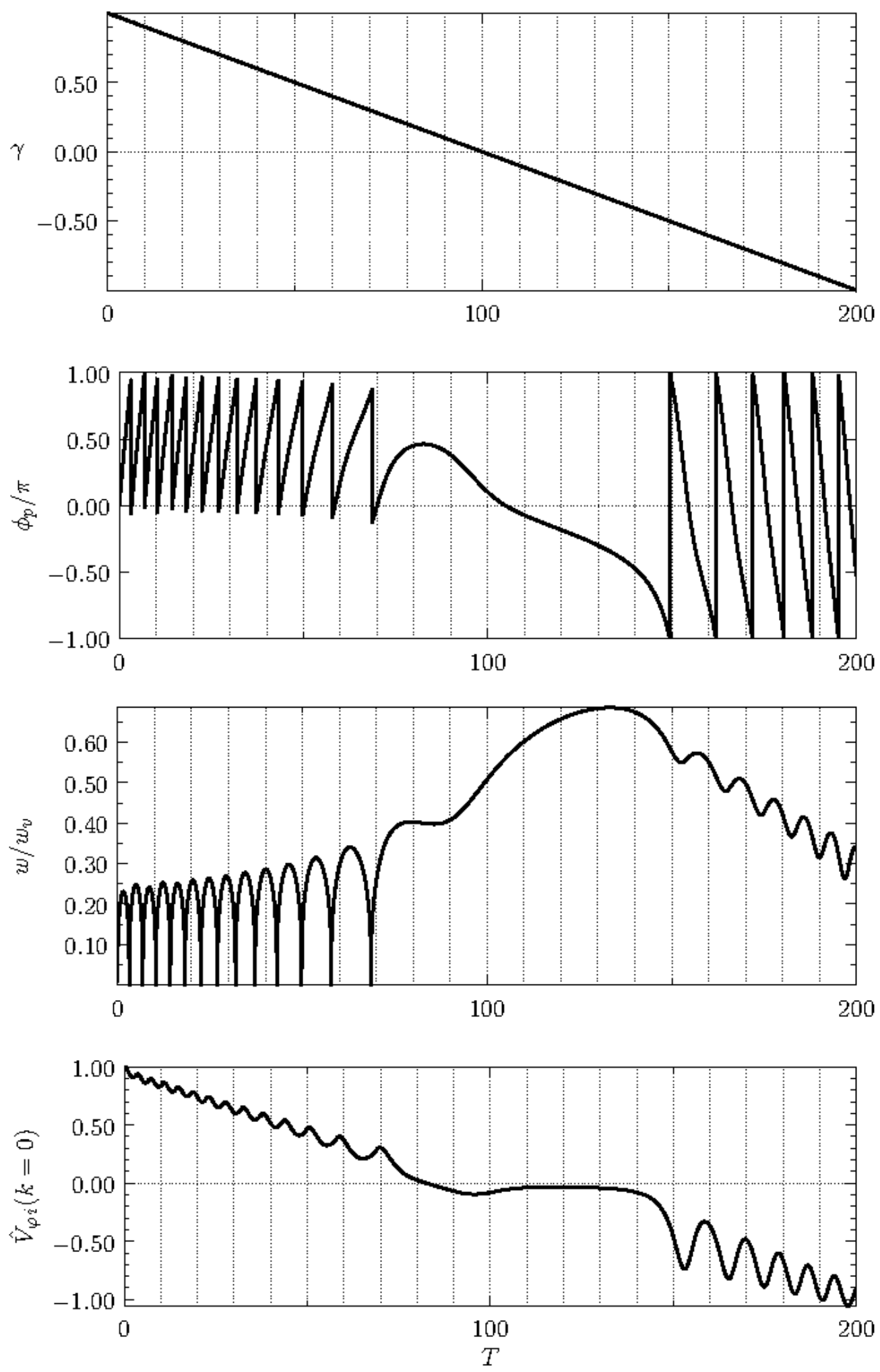

FIG. 8. Numerical solution of Eqs. (7) and (8) with $\lambda_{R}=100.0, b_{v}=0.6$, and $\bar{\nu}=0.1$. In order from the top to the bottom, the panels show the normalized natural frequency, the island helical phase, the ratio of the island width to the vacuum island width, and the normalized toroidal ion velocity inside the island separatrix. 

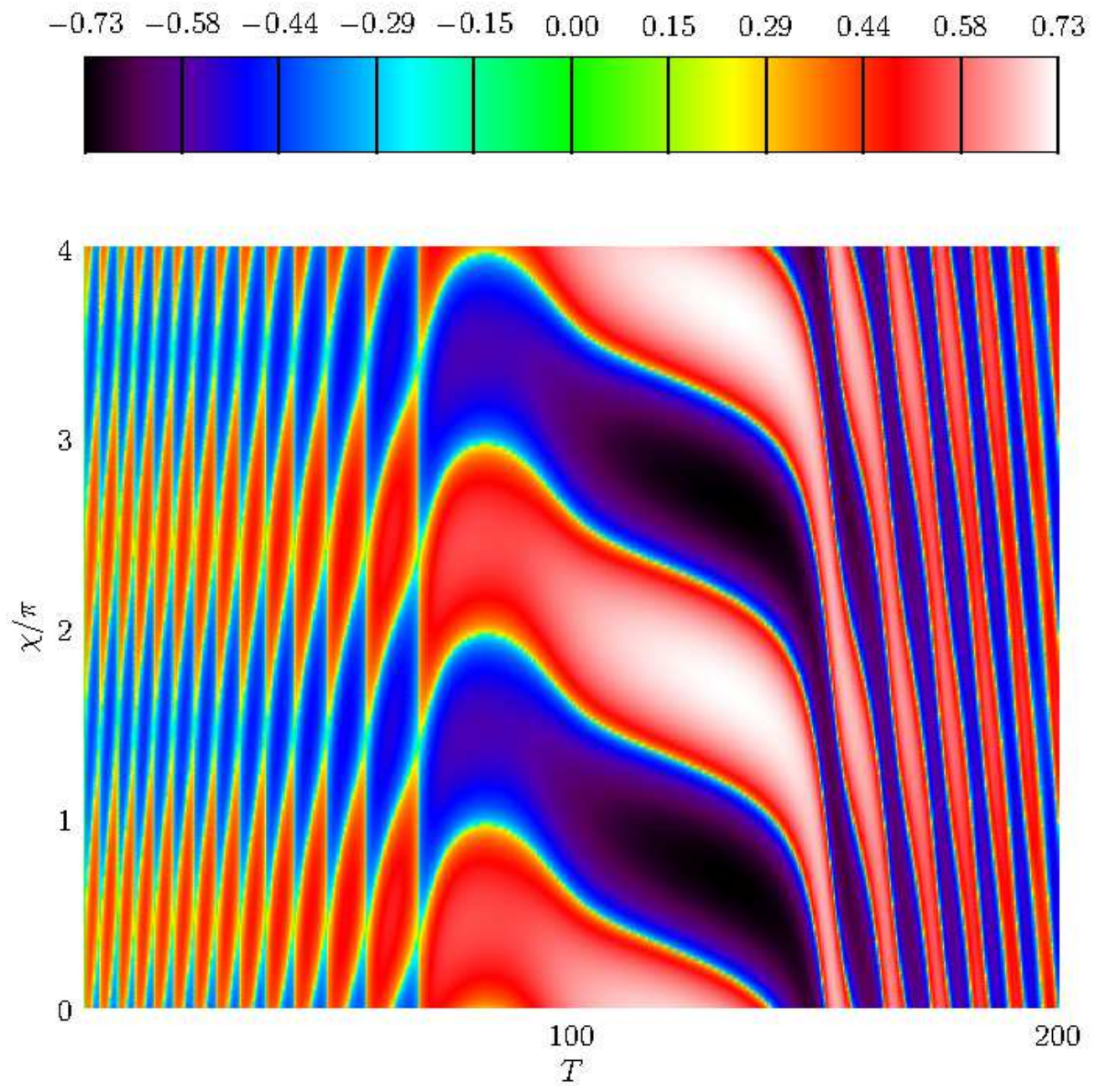

FIG. 9. Simulated Mirnov data for the case shown in Fig. 8 . 